\title{
Deceptions of Early Repolarisation Syndrome
}

\author{
Dr Venkatesh Tekur Krishnamurthy ${ }^{1}$ Dr Sriarun Tekur Venkatesh ${ }^{2}$ \\ ${ }^{l}$ consultant Interventional Cardiologist Apollo Hospitals Bannerghatta Road Bangalore \\ ${ }^{2}$ intern Jss Hospital Mahathma Gandhi Road, Mysuru, Karnataka
}

\begin{abstract}
Early repolarisation pattern on ECG when a patient presents with chest pain needs careful evaluation to exclude coronary artery disease. The Authors report here a deceptive ECG pattern of early repolarisation in a patient with severe chest pain which necessitated coronary angiogram revealing normal epicardial coronary arteries. Subsequently, patient developed the typical ECG pattern of early repolarisation. Awareness of the deceptive ECG variant of early repolarisation pattern can avoid invasive coronary angiograms in these patients.
\end{abstract}

Keywords: Early Repolarisation Ecg Coronary Angiogram

Key messages:Awareness of the deceptive ECG variant of early repolarisation pattern can avoid invasive coronary angiograms in these patients.

\section{Introduction}

The term early repolarization (ER), also known as "J-waves" or "J-point elevation," has long been used to characterize a QRS-T variant on the electrocardiogram $(\mathrm{ECG})^{1}$. Numerous reports have suggested association between ER and an increased risk for arrhythmic death and idiopathic ventricular fibrillation $(\mathrm{VF})^{2-4}$. ER syndrome is diagnosed in the presence of J-point elevation $\geq 1 \mathrm{~mm}$ in $\geq 2$ contiguous inferior and/or lateral leads of a standard 12-lead ECG in a patient resuscitated from otherwise unexplained VF/polymorphic VT. ER syndrome can be diagnosed in an Sudden cardiac death victim with a negative autopsy and medical chart review with a previous ECG demonstrating J-point elevation $\geq 1 \mathrm{~mm}$ in $\geq 2$ contiguous inferior and/or lateral leads of a standard 12-lead ECG. ER pattern can be diagnosed in the presence of J-point elevation $\geq 1 \mathrm{~mm}$ in $\geq 2$ contiguous inferior and/or lateral leads of a standard 12-lead ECG ${ }^{5}$. The Authors here describe a patient who presented with chest pain and a deceptive variant of early repolarisation pattern on ECG which required a coronary angiogram to exclude coronary artery disease.

\section{Case History}

A 32 year male presented to the emergency department with sudden onset of retrosternal chest pain of one hour duration. He also had a family history of coronary artery disease with his father having suffered acute myocardial infarction at 54 years of age. ECG showed ST segments elevation and the T wave inversions in many leads, especially V3 and V4.(FIGURE 1)

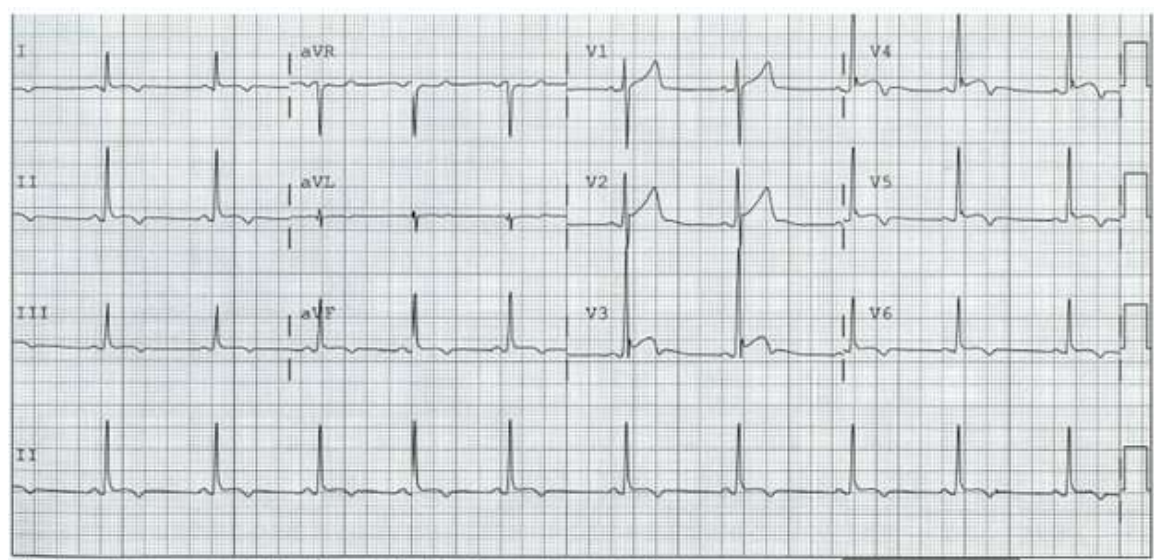

Figure 1: Showing St Segment Elevation And T Wave Inversions In Many Leads.

After preliminary investigations, patient was quickly taken to the cath lab and a coronary angiogram was performed via right radial approach. It revealed normal coronary arteries. About two hours later, a ECG was taken again which showed the typical Early repolarisation pattern.(FIGURE 2). 


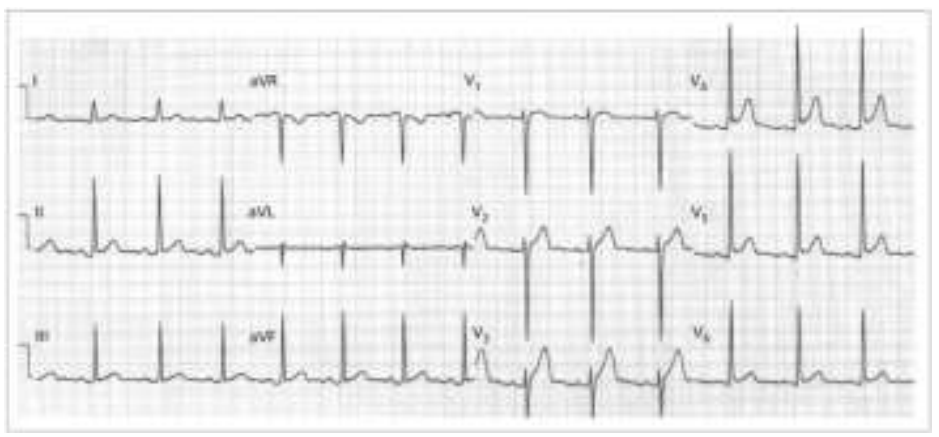

Figure 2: Typical Early Repolarisation Pattern After One Hour.

\section{Discussion}

Early Repolarisation[ER] is an ECG finding. Two terms, distinguished by the presence or absence of symptomatic arrhythmias, have been used to describe patients with this ECG finding. The ER pattern describes the patient with appropriate ECG findings in the absence of symptomatic arrhythmias. The ER syndrome applies to the patient with both appropriate ECG findings and symptomatic arrhythmias. Persons with either the ER pattern or ER syndrome can have identical findings on surface ECG. However, the mere presence of ER pattern on ECG should not lead to a classification of ER syndrome in the absence of symptoms or documented ventricular fibrillation (VF). On the ECG, ER is defined as either a sharp well-defined positive deflection or notch immediately following a positive QRS complex at the onset of the ST segment or the presence of slurring at the terminal part of the QRS complex (since the J-wave or J-point elevation may be hidden in the terminal part of the QRS complex, resulting in the slurring of the terminal QRS complex). Most literature defines ER as being present on the ECG when there is J-point elevation of $\geq 0.1 \mathrm{mV}$ in two adjacent leads with either a slurred or notched morphology. ${ }^{6}$. Based on data associating arrhythmic risk with spatial distribution of ER, a classification scheme has been proposed in early repolarisation ${ }^{7}$. Type 1 is associated with ER in the lateral precordial leads. This form is common among healthy male athletes and is thought to be largely benign.Type 2 is associated with ER in the inferior or inferolateral leads and is associated with a moderate level of risk.Type 3 is associated with ER globally in the inferior, lateral, and right precordial leads, and appears to be associated with the highest relative risk, though the absolute risk of sudden death remains small.Type 4 , or Brugada syndrome, is marked by J-wave/point elevation in the right precordial leads.The ER pattern is not always identified on routine ECG due to the intermittent nature of ER ${ }^{8}$. As an example, among 542 persons with baseline ER who underwent repeat ECG examination five years later, ER $(\geq 0.1 \mathrm{mV})$ was not observed in approximately 20 percent $^{6}$. The perception that ER is a benign finding devoid of clinical significance has changed. Large population studies have shown the presence of ER in the inferior leads on surface ECG is associated with an increased risk for death from cardiac causes as well as all-cause mortality ${ }^{9,10}$. Patients with ECG findings of the ER pattern appear to be at an increased risk of VF in the event of a myocardial infarction/ischemia ${ }^{11}$.The ER pattern in the inferior leads has been associated with an increased risk of sudden cardiac death in patients with congestive heart failure ${ }^{12}$. As is seen in ER, there is J-point elevation with resultant ST segment elevation in patients with acute pericarditis. Symptom presentation is markedly different in the two conditions. Unlike ER, most patients with acute pericarditis have ST elevations diffusely in most or all limb and precordial leads. Additionally, patients with acute pericarditis often have deviation of the PR segment, which is not present in ER. While patients with acute myocardial injury due to ST elevation myocardial infarction (STEMI) can initially have elevation of the J-point with concave ST segment elevation, the ST segment elevation typically becomes more pronounced and convex (rounded upward) as the infarction persists. However, the primary distinguishing factor between ER and acute myocardial injury is the presence of clinical symptoms such as chest pain or dyspnea. The Authors present here an interesting varient of early repolarisation ECG pattern. The same has been mentioned by Chou TC,and Ramaiah $\mathrm{LS}^{13}$. They describe it as a normal variant. The similarity between this entity and the early repolarization pattern as a normal variant is that there is a notch at the junction ${ }^{14}$. However, the two entities are different in that the ST segments are coved and the $\mathrm{T}$ waves are inverted here, whereas in the early repolarization pattern, the ST segments are concave and the $\mathrm{T}$ waves are upright. Awareness of the variant pattern of early repolarisation ECG can avoid invasive coronary angiograms in these subset of patients.

\section{References}

[1]. Rautaharju PM, Surawicz B, Gettes LS, et al. AHA/ACCF/HRS recommendations for the standardization and interpretation of the electrocardiogram: part IV: the ST segment, T and U waves, and the QT interval: a scientific statement from the American Heart Association Electrocardiography and Arrhythmias Committee, Council on Clinical Cardiology; the American College of Cardiology Foundation; and the Heart Rhythm Society: endorsed by the International Society for Computerized Electrocardiology. Circulation 2009; 119:e241. 
[2]. Sinner MF, Reinhard W, Müller M, et al. Association of early repolarization pattern on ECG with risk of cardiac and all-cause mortality: a population-based prospective cohort study (MONICA/KORA). PLoS Med 2010; 7:e1000314.

[3]. Haïssaguerre M, Derval N, Sacher F, et al. Sudden cardiac arrest associated with early repolarization. N Engl J Med 2008; 358:2016.

[4]. Abe A, Ikeda T, Tsukada T, et al. Circadian variation of late potentials in idiopathic ventricular fibrillation associated with J waves: insights into alternative pathophysiology and risk stratification. Heart Rhythm 2010; 7:675.

[5]. Priori SG, Wilde AA, Horie M, et al. HRS/EHRA/APHRS Expert Consensus Statement on the Diagnosis and Management of Patients with Inherited Primary Arrhythmia Heart Rhythm 2013; 10:1932.

[6]. Tikkanen JT, Anttonen O, Junttila MJ, et al. Long-term outcome associated with early repolarization on electrocardiography. N Engl J Med 2009; 361:2529.

[7]. Antzelevitch C, Yan GX, Viskin S. Rationale for the use of the terms J-wave syndromes and early repolarization. J Am Coll Cardiol 2011; 57:1587.

[8]. Derval N, Simpson CS, Birnie DH, et al. Prevalence and characteristics of early repolarization in the CASPER registry: cardiac arrest survivors with preserved ejection fraction registry. J Am Coll Cardiol 2011; 58:722.

[9]. Haruta D, Matsuo K, Tsuneto A, et al. Incidence and prognostic value of early repolarization pattern in the 12-lead electrocardiogram. Circulation 2011; 123:2931.

[10]. Olson KA, Viera AJ, Soliman EZ, et al. Long-term prognosis associated with J-point elevation in a large middle-aged biracial cohort: the ARIC study. Eur Heart J 2011; 32:3098.

[11]. Patel $\mathrm{RB}$, Ilkhanoff $\mathrm{L}, \mathrm{Ng} \mathrm{J}$, et al. Clinical characteristics and prevalence of early repolarization associated with ventricular arrhythmias following acute ST-elevation myocardial infarction. Am J Cardiol 2012; 110:615.

[12]. Furukawa Y, Yamada T, Morita T, et al. Early repolarization pattern associated with sudden cardiac death: long-term follow-up in patients with chronic heart failure. J Cardiovasc Electrophysiol 2013; 24:632.

[13]. Chou TC, Ramaiah LS. Electrocardiography in Clinical Practice: Adult and Pediatric. 4th ed. Philadelphia, PA: WB Saunders Co.; 1996: 190 .

[14]. Wang K. Atlas of Electrocardiography. Philadelphia, PA: Jaypee Brothers Medical Publishing; 2013:226. 